\title{
Measurement of Mitral Valve Area By Direct Three Dimensional Planimetry Compared To Multiplanar Reconstruction In Patients With Rheumatic Mitral Stenosis
}

\section{Mehrnoush Toufan Tabrizi}

Tabriz university of medical science

Haniyeh Faraji Azad

Tabriz university of medical science

Naser Khezerlouy-Aghdam ( $\square$ nkhezerlou90@gmail.com )

Islamic Azad University https://orcid.org/0000-0001-5446-1492

Hanieh Sakha

Tabriz university of medical sciences

\section{Research Article}

Keywords: measurements, three-dimensional, Transmitral, Intraclass

Posted Date: October 21st, 2021

DOl: https://doi.org/10.21203/rs.3.rs-984280/v1

License: (c) (i) This work is licensed under a Creative Commons Attribution 4.0 International License.

Read Full License

Version of Record: A version of this preprint was published at The International Journal of Cardiovascular Imaging on January 19th, 2022. See the published version at https://doi.org/10.1007/s10554-022-025230 . 


\section{Abstract}

Background: Mitral valve area (MVA) measurement by three-dimensional transesophageal echocardiography (3D-TEE) has a crucial role in the evaluation of mitral stenosis (MS) severity. Threedimensional direct (3D-direct) planimetry has been proposed as a new technique to measure mitral valve area. This study aimed to compare the 3D-direct mitral valve planimetry to conventional threedimensional multiplanar reconstruction (3D-MPR) in severe mitral stenosis (MS) using 3D-TEE.

Methods: 149 patients with severe MS who were referred for percutaneous transmitral commissurotomy (PTMC), prospectively recruited. All patients underwent 2D transthoracic echocardiography (2D-TTE) and 3D-TEE in a single session before PTMC. During 2D-TTE planimetry, pressure half time (PHT), and proximal isovelocity surface area (PISA) were applied to measure the MVA. Transmitral mean pressure gradient (MPG) was measured. During 3D-TEE, MVA planimetry was carried out with both 3D-direct and 3D-MPR methods. 3D-direct was applied from both atrial and ventricular views. The consistency of MVA measurements with 3D-direct, 3D-MPR, and 2D-TTE methods was statistically investigated.

Results: Our sample consisted of 109 (73.2\%) women and 40 (26.8\%) men. The mean age was $51.75 \pm$ 9.81 years. The agreement between 3D-direct and 3D-MPR planimetry was significant and moderate (0.99 $\pm 0.29 \mathrm{~cm}^{2}$ vs. $1.12 \pm 0.26 \mathrm{~cm}^{2}$, Intraclass Correlation $=0.716, p$ value $\left.=0.001\right)$. The accuracy of the 3Ddirect method reduced significantly compared to the MPR method at MVA $>1.5 \mathrm{~cm}^{2}$. The maximum difference between two methods was observed in cases with MVAs larger than $1.5 \mathrm{~cm}^{2}$. MVA measured with the 3D-MPR method was significantly correlated with a 2D-TTE method, with a moderate agreement (Intraclass Correlation $=0.644, \mathrm{p}$ value $=0.001)$. Also, 2D-TTE and 3D-direct TEE techniques yielded significantly consistent measurements of the MVA $\left(1.06 \pm 0.026 \mathrm{~cm}^{2}\right.$ vs. $0.99 \pm 0.29 \mathrm{~cm}^{2}$, Intraclass Correlation $=0.787$, $p$ value $=0.001)$; however, with a slight overestimation of the MVA by the former with a net difference of $0.06 \pm 0.013 \mathrm{~cm}^{2}$. Mitral valve pressure gradient (MPG) had no significant correlation with planimetry results. A significant inverse correlation was seen between the MVA and pulmonary arterial systolic pressure.

Conclusion: 3D-direct planimetry has an acceptable agreement with 3D-MPR planimetry at MVA less than $1.5 \mathrm{~cm}^{2}$, but their correlation decreases significantly at MVA above $1.5 \mathrm{~cm}^{2}$. 3D-direct planimetry underestimates MVA compared to 3D-MPR, especially at MVA above $1.5 \mathrm{~cm}^{2}$. The 2D-TTE planimetry has generally acceptable accuracy, but its correlation to the 3D-TEE methods is significantly reduced in cases with moderate to severe MS (i.e. MVA>1.0cm2).

\section{Introduction}

Rheumatic heart disease (RHD) remains the most common reason for mitral stenosis (MS), although its prevalence has decreased drastically in the developed countries $(1,3)$. In the developing countries, RHD is still the major cause of MS (e.g. $97 \%$ of cases in India) (2). Mitral valve area (MVA) planimetry using twodimensional transthoracic echocardiography (2D-TTE) is a common accurate method for evaluating the 
severity of rheumatic mitral stenosis (MS) and planning for management $(4,5)$. Although the 2D-TTE measurement of MVA is considered a sophisticated method (6), expertise and proper echocardiographic windows are required to perform it precisely on the tip of the mitral valve leaflets $(\mathrm{MV})$ in a well-oriented plane (7). In recent years, technological advances in 3D echocardiography have made possible more realistic visualization and investigation of MVA measurement. This technique improved the accuracy of the planimetry in non-experienced operators significantly $(7,8)$. 3D-TEE has an impressive diagnostic value in the evaluation of mitral valve commissures (9). Real-time three-dimensional transesophageal echocardiography (3D-TEE) produced excellent images with high resolutions of the MVA $(8,10)$. 3D-direct planimetry has been proposed as a new planimetry method of MVA. Recent small studies showed acceptable agreement between 3D-direct and 3D-MPR planimetry methods with some discrepancies which are attributed to the differences in measurement of major diameter of mitral valve $(11,12)$. Considering the saddle-like shape of the mitral valve and its possible effect on planimetry, we aimed to compare the results of 3D- direct and 3D-MPR planimetry methods. We compared the results of these two methods at the valve area below $1 \mathrm{~cm}^{2}, 1-1.5 \mathrm{~cm}^{2}$ and above $1.5 \mathrm{~cm}^{2}$ in large numbers of patients with MS to assess the role of mitral valve shape on planimetry. Moreover, we evaluated the association between 2D-TTE and 3D-TEE planimetry results, with a consideration of their moderate to good agreement which is addressed at some previously published studies (13).

\section{Methods}

\section{Subjects and the design of the study}

This is a cross-sectional, observational study. All adult patients (18-80 years old) with severe MS, who were initially candidates for PTMC, were recruited. The inclusion period was from April 2017 to March 2019. The study took place at Shahid Madani Heart Hospital, which is the tertiary heart center in Tabriz, Iran. All patients underwent 2D-TTE and 3D-TEE for measurement of the MVA in a single inpatient session on the same day before PTMC. The exclusion criteria were the history of PTMC during last month and contraindication of TEE (e.g. esophageal disorders). Patients with LA, LAA clot or progressive MS (i.e. MVA>1.5 $\mathrm{cm}^{2}$ ) based on 3D planimetry results were excluded from subsequent PTMC.

\section{D -TTE}

2D-TTE images were obtained using a Philips EPIQ 7C ultrasound machine with an X5-1 matrix probe (Philips Co., Netherlands). In direct planimetry, the MVA was measured by the operator after adjusting the setting to an optimized view. The smallest optimal mitral valve orifice was captured on leaflet tips through the parasternal short-axis view in mid-diastole during the maximal opening at smallest orifice dimension (5). Furthermore, MVA was estimated through pressure half time (PHT) and proximal isovelocity surface area (PISA) models. Transmitral mean pressure gradient (MPG), and peak mitral flow velocity were measured, and MR severity was assessed based on American Society of Echocardiography guideline recommendations (14). LV ejection fraction (LVEF), LA volume index (LAVI), pulmonary artery 
systolic pressure (based on tricuspid regurgitation peak pressure gradient) were measured. Concomitant aortic, tricuspid, and pulmonary valve regurgitation and/or stenosis were evaluated.

\section{D -TEE}

A 2-7 MHz, real-time 3D-TEE X matrix-array transducer (Philips EPIQ 7C ultrasound machine) was used to obtain the 3D images. After optimizing the gain (generally at mid-range, 50 units), compression controls, and time gain compensation over four consecutive heartbeats at sinus rhythm, a 3D Zoom data set was acquired at $0,45,75$, and 120 degrees. At atrial fibrillation, single beat acquisition was applied and images with stitch artifacts were discarded. At the 3D-MPR method, one 2D-cut plane was place on MV leaflets tips, and other planes were placed perpendicularly. Multiple slice mode (islice method) was applied parallel to MV leaflets tips to determine the narrowest MV orifice. At the 3D-direct method, all 3D data sets were cropped. In this method, planimetry was applied at en face MV orientation from both left atrial and left ventricular sides. Planimetry at both 3D-direct and 3D-MPR methods was performed on MV leaflets tips in mid diastole during the maximal opening, at the smallest orifice dimension $(11,12,13)$. All measurements were done with an expert echocardiographist and were subsequently reviewed by a second echocardiographist. In cases with suboptimal quality of the 3D images, multiple attempts were made to achieve the optimal measurement.

\section{Statistical Analysis}

Statistical analysis was performed using the IBM SPSS Statistics for Windows, Version 22.0. Continuous variables were expressed as the mean \pm SD. Categorical variables were presented in frequencies and percentages. Mean values for two variables were compared using a paired T-test and Chi-squared test in normal distributions and Mann-Whitney $U$ test in non-normal distributions. $P$ values $<0.05$ were considered statistically significant. For further analysis, MVA was categorized into 3 subgroups including MVA $<1.0 \mathrm{~cm}^{2}, 1.0-1.5 \mathrm{~cm}^{2}$, and $>1.5 \mathrm{~cm}^{2}$. The agreement of measured MVA by 2D-TTE and 3D-TEE was evaluated by the two-way mixed intra-class correlation coefficient (ICC), Pearson correlation coefficient (R), and the Bland-Altman method.

\section{Ethics}

The research protocol was approved by the institutional review board of research ethics at Tabriz University of Medical Sciences, Iran. The procedure and patient rights were explained to the subjects verbally, and written informed consent was obtained from all participants.

\section{Results}

One hundred forty-nine subjects were enrolled in this study. Clinical characteristics of patients are summarized in Table.1. The sample population was composed of $109(73.2 \%)$ female and $40(26.8 \%)$ male patients with an average age of $51.75 \pm 9.81$ years. More than half of the patients $(55.0 \%)$ had sinus rhythm, while the remainder of them had atrial fibrillation. Most of the included patients were 
symptomatic, with dyspnea being the most common complaint (89.9\%). Tricuspid insufficiency was present in $96.6 \%$ of patients, which was followed by mitral regurgitation in $89.3 \%$, and aortic regurgitation in $74.5 \%$.

The echocardiographic results of all patients with different 2D-TTE and 3D-TEE methods are summarized in Table.2. The mean MVA measured by 3D-direct method was $0.99 \pm 0.29 \mathrm{~cm}^{2}$, while MVA by 3D-MPR method was $1.12 \pm 0.26 \mathrm{~cm}^{2}$. MVA measurement in the 3D-direct method overally was in significant concordance with 3D-MPR method with a good agreement (Intraclass Correlation $=0.716$, P-value $=0.001)$. MVA measured by 3D-direct through LA and LV views was statistically in concordance with an excellent agreement (Intraclass Correlation $=0.964$, p value $=0.001$ ). The average MVA in 2D-TTE was $1.06 \pm 0.026 \mathrm{~cm}^{2}$. MVA measurement in the 2D-TTE had a significant correlation with 3D-MPR method with a moderate agreement (Intraclass Correlation= 0.644, P-value $=0.001$ ), while MVA measurement in the 2D-TTE had significant consistency with 3D-direct method with a good agreement. (Intraclass Correlation $=0.787$, P-value $=0.001)$ (Figure 1). Comparing the different measurement methods, it was found out that, on average, MVA measured by 3D-direct was $0.12 \pm 0.003 \mathrm{~cm}^{2}$ less than that of 3D-MPR and $0.06 \pm 0.013 \mathrm{~cm}^{2}$ less than that of 2D-TTE. The MVA measured by 3D-MPR method was $0.06 \pm 0.019$ larger than that of 2D-TTE (Figure 2). The left atrium was enlarged severely in most cases and the mean LAVI was $82.39 \pm 1.34 \mathrm{ml} / \mathrm{m}^{2}$. Pulmonary artery systolic pressure increased modestly in most patients and mean SPAP was $44.6 \pm 14.56 \mathrm{mmHg}$. The mean LVEF was $52 \% \pm 4 \%$.

The echocardiographic findings of all the patients were analyzed in three subgroups of MVA (i.e. $<1 \mathrm{~cm}^{2}$, $1-1.5 \mathrm{~cm}^{2},>1.5 \mathrm{~cm}^{2}$ ) (Table 3). The analysis of the subgroups of MVA between 3D-direct and 3D-MPR TEE methods showed significant correlation with a moderate agreement at MVA less than $1 \mathrm{~cm}^{2}$, weak agreement at MVA between $1-1.5 \mathrm{~cm}^{2}$ and no significant agreement at MVA above $1.5 \mathrm{~cm}^{2}$. Further analysis of the three categories of MVA between 2D and 3D-direct methods showed good agreement at MVAs below $1 \mathrm{~cm}^{2}$, moderate agreement between $1 \mathrm{~cm}^{2}$ and $1.5 \mathrm{~cm}^{2}$, and less agreement in MVA more than $1.5 \mathrm{~cm}^{2}$. The analysis of MVA between 2D-TTE and 3D-MPR methods showed significant correlation with a moderate agreement at MVA less than $1 \mathrm{~cm}^{2}$, weak agreement at MVA between $1-1.5 \mathrm{~cm}^{2}$, and no significant agreement at MVA above $1.5 \mathrm{~cm}^{2}$ 
Table 1

Baseline characteristics of the patients. PTMC, percutaneous transmitral commissurotomy

\begin{tabular}{|c|c|c|}
\hline \multicolumn{2}{|l|}{ Subjects(n) } & 149 \\
\hline \multicolumn{2}{|l|}{ Age mean \pm sd } & $51.75 \pm 9.81$ \\
\hline \multicolumn{2}{|l|}{ Female / Male ratio } & $109(73.1 \%) / 40(26.8 \%)$ \\
\hline \multicolumn{2}{|l|}{ Atrial fibrillation $\mathrm{n}(\%)$} & $66(44.3 \%)$ \\
\hline \multicolumn{2}{|l|}{ History of PTMC n(\%) } & $34(22.8 \%)$ \\
\hline \multicolumn{2}{|l|}{ Dyspnea n(\%) } & $134(89.9 \%)$ \\
\hline \multirow[t]{6}{*}{ Mitral regurgitation n(\%) } & Total & $133(89.3 \%)$ \\
\hline & Trivial & $5(3.4 \%)$ \\
\hline & Mild & $16(10.7 \%)$ \\
\hline & Mild to Moderate & $60(40.3 \%)$ \\
\hline & Moderate & $37(24.7 \%)$ \\
\hline & Severe & $15(10.1 \%)$ \\
\hline \multirow[t]{6}{*}{ Aortic regurgitation n(\%) } & Total & $111(74.5 \%)$ \\
\hline & Trivial & $8(5.4 \%)$ \\
\hline & Mild & $5(3.4 \%)$ \\
\hline & Mild to Moderate & $38(25.5 \%)$ \\
\hline & Moderate & $43(28.9 \%)$ \\
\hline & Severe & $17(11.3 \%)$ \\
\hline \multirow[t]{4}{*}{ Aortic stenosis n(\%) } & Total & $13(8.4 \%)$ \\
\hline & Mild & $9(6 \%)$ \\
\hline & Moderate & $2(1.3 \%)$ \\
\hline & Severe & $2(1.3 \%)$ \\
\hline \multirow[t]{5}{*}{ Tricuspid regurgitation $\mathrm{n}(\%)$} & Total & $141(96.6 \%)$ \\
\hline & Trivial & $5(3.4 \%)$ \\
\hline & Mild & $7(4.7 \%)$ \\
\hline & Mild to Moderate & $67(45 \%)$ \\
\hline & Moderate & $42(28.2 \%)$ \\
\hline
\end{tabular}




\begin{tabular}{|lll|}
\hline Subjects(n) & & 149 \\
\hline Tricuspid stenosis $\mathrm{n}(\%)$ & Severe & $20(13.4 \%)$ \\
& Total & $1(0.7 \%)$ \\
& Trivial & 0 \\
\hline Mild & 0 \\
\hline Mild to Moderate & 0 \\
\hline Moderate & 0 \\
& Severe & $1(0.7 \%)$ \\
\hline
\end{tabular}

Table 2

MVA is measured through different methods. All mitral valve area measurements are reported in $\mathrm{cm}^{2}$

\begin{tabular}{|ll|}
\hline Mean pressure gradient $(\mathrm{mmHg})$ & $\mathbf{8 . 3 8} \pm \mathbf{4 . 7 1}$ \\
\hline Peak velocity $(\mathrm{m} / \mathrm{s})$ & $2.06 \pm 0.44$ \\
\hline MVA by 3D - direct $\left(\mathrm{cm}^{2}\right)$ & $0.99 \pm 0.29$ \\
\hline MVA through LA view $\left(\mathrm{cm}^{2}\right)$ & $0.98 \pm 0.29$ \\
\hline MVA through LV view $\left(\mathrm{cm}^{2}\right)$ & $0.99 \pm 0.29$ \\
\hline MVA by 3D -MPR $\left(\mathrm{cm}^{2}\right)$ & $1.12 \pm 0.26$ \\
\hline MVA by 2D planimetry $\left(\mathrm{cm}^{2}\right)$ & $1.06 \pm 0.45$ \\
\hline MVA by 2D PHT(cm $\left.{ }^{2}\right)$ & $1.13 \pm 0.3$ \\
\hline MVA by PISA (cm $\left.{ }^{2}\right)$ & $1.00 \pm 0.37$ \\
\hline Pulmonary artery systolic pressure $(\mathrm{mmHg})$ & $44.6 \pm 14.56$ \\
\hline LA Volume Index (ml/m²) & $82.39 \pm 1.34$ \\
\hline Mitral valve score $($ Wilkins score) & $9.4 \pm 1.34$ \\
\hline Left Ventricle Ejection fraction $)$ & $52 \% \pm 4 \%$
\end{tabular}

LA left atrium; LV left ventricle; MPR multiplanar reconstruction; MVA mitral valve area; PHT pressure half time; PISA proximal isovelocity surface area. 
Table 3

Number of patients in different subgroups of MVA. MPR multiplanar reconstruction; MVA mitral valve area; TTE transthoracic echocardiography.

\begin{tabular}{|llll|}
\hline \multirow{4}{*}{} & \multicolumn{3}{l}{ MVA $\left(\mathrm{cm}^{2}\right)$} \\
\cline { 2 - 4 } & $<1$ & $1-1.5$ & $\geq 1.5$ \\
\hline 3D-direct & $52.3 \%$ & $43 \%$ & $4 \%$ \\
\hline 3D-MPR & $49 \%$ & $34 \%$ & $16 \%$ \\
\hline 2D-TTE & $34.9 \%$ & $58.4 \%$ & $4 \%$ \\
\hline
\end{tabular}

(Table 4). Most of the patients had a mean pressure gradient between 5 to $10 \mathrm{mmhg}$ (45.0\%). There was no significant correlation between MPG and MVA measured by different methods including 2D-TTE, 3Ddirect, 3D-MPR, PISA and PHT (P-value >0.05). The systolic PAP had a significant inverse correlation with MVA in all measurement methods including 3D-direct ( $P$-value $=0.001, r=0.358), 2 D-T T E$ (P-value $=$ $0.001, r=-0.241)$, and 3D-MPR (P-value $=0.001, r=0.369)$. Interestingly, there was no significant relationship between severity of LA enlargement and MVA in any of the measurement methods (P-value $>0.05)$.

Table 4

Comparing correlation of 2D-TTE, 3D-direct and 3D-MPR planimetry methods at different subgroups of MVA (i.e. $<1 \mathrm{~cm}^{2}, 1-1.5 \mathrm{~cm}^{2},>1.5 \mathrm{~cm}^{2}$ ).

\begin{tabular}{|c|c|c|c|c|c|c|}
\hline \multicolumn{3}{|c|}{ Compared Groups } & \multirow{3}{*}{$\begin{array}{l}\text { Intraclass Correlation } \\
0.779\end{array}$} & \multicolumn{2}{|c|}{$95 \%$ Confidence Interval } & \multirow[t]{2}{*}{$P$ value } \\
\hline & & & & Lower Bound & Upper Bound & \\
\hline \multirow[t]{2}{*}{ A } & \multirow{3}{*}{$\begin{array}{l}\text { 2D-TTE } \\
\text { and } \\
\text { 3D Direct }\end{array}$} & $<1 \mathrm{~cm}^{2}$ & & 0.673 & 0.854 & 0.001 \\
\hline & & $1-1.5 \mathrm{~cm}^{2}$ & 0.605 & 0.418 & 0.743 & 0.001 \\
\hline & & $\geq 1.5 \mathrm{~cm}^{2}$ & -0.398 & -.0365 & 0.841 & 0.144 \\
\hline \multirow[t]{3}{*}{ B } & \multirow{3}{*}{$\begin{array}{l}\text { 2D-TTE } \\
\text { and } \\
\text { 3D -MPR }\end{array}$} & $<1 \mathrm{~cm}^{2}$ & 0.674 & 0.535 & 0.778 & 0.001 \\
\hline & & $1-1.5 \mathrm{~cm}^{2}$ & 0.309 & 0.418 & 0.743 & 0.007 \\
\hline & & $\geq 1.5 \mathrm{~cm}^{2}$ & -0.457 & -.0365 & 0.841 & 0.781 \\
\hline \multirow[t]{3}{*}{ C } & \multirow{3}{*}{$\begin{array}{l}\text { 3D-Direct } \\
\text { And } \\
\text { 3D -MPR }\end{array}$} & $<1 \mathrm{~cm}^{2}$ & 0.665 & 0.524 & 0.770 & 0.001 \\
\hline & & $1-1.5 \mathrm{~cm}^{2}$ & 0.240 & -0.011 & 0.462 & 0.030 \\
\hline & & $\geq 1.5 \mathrm{~cm}^{2}$ & -0.139 & -0.854 & 0.785 & 0.604 \\
\hline
\end{tabular}


The inter-observer agreement in the measurement of MVA by 3-MPR between two expert echocardiogeaphists was 0.745 with P-value $=0.001$.

\section{Discussion}

The results of our study with a relatively large sample size indicated that MVA measurement by two different 3D-direct and 3D-MPR planimetry methods had consistent results, with moderate to excellent agreement. Overlay MVA measured by the 3D-direct method is less than 3D-MPR, and mitral stenosis severity is overestimated by 3D-direct dominantly at MVA above $1.5 \mathrm{~cm}^{2}$. Our subgroup analysis revealed that although there was a significant correlation between 3D-direct and 3D-MPR in very severe MS (i.e. $M V A<1 \mathrm{~cm}^{2}$ ), no significant correlation was observed in cases with progressive (moderate) MS (i.e. $M V A>1.5 \mathrm{~cm}^{2}$ ). 3D-MPR is the most accurate method for planimetry and can theoretically produce reliable measurements by delineating the mitral valve orifice. The discrepancy between the 3D- MPR and 3D-direct planimetry methods in moderate MS is mainly due to the limitation of the latter in obtaining appropriate en face plane to evaluate the entire MV in a single view because of its saddle-like shape and relatively pliable leaflets at progressive (moderate) MS. (Fig. 3) In practice, we need to frequently tilt the $3 \mathrm{D}$ volume image of $\mathrm{MV}$, so that we can see the entire MV orifice in the patients with progressive MS. So using a single en face plane during 3D-direct planimetry will underestimate MVA significantly.

Considering the higher accuracy of the MPR technique, it seems that in the case of patients with progressive (moderate) MS, it is better to measure the MVA by 3D-MPR method instead of 3D-direct. 3DMPR is a 3D data set processing that combines image reconstruction and multiplanar cropping to identify the narrowest orifice of the valve (Fig. 3). Recently, Zhong et al., by comparing 3D-direct and 3DMPR methods in the case of patients with MS demonstrated that while MVA measured by 3D-direct was significantly lower than that obtained by 3D-MPR (12). The underestimation of MVA by 3D-direct was also reported by other studies $(13,15)$. Our study confirms and extends the findings of previous studies as we compared 3D-direct and MPR techniques in different severities of MS.

MVA measured by 2D-TTE and various 3D-TEE methods had consistent results with moderate to excellent agreement. The MVA measured by 2D method was on average lower than that measured by 3D-MPR method and, in general, 2D method overestimated MS severity. Comparing the three subgroups of MVA (MVA $<1 \mathrm{~cm}^{2}$, MVA between 1-1.5 $\mathrm{cm}^{2}$ and MVA $>1.5 \mathrm{~cm}^{2}$ ) measured by 3D-direct, 3D-MPR and 2D methods showed that there is a good agreement between 2D and different 3D methods at MVAs below 1 $\mathrm{cm}^{2}$, moderate agreement at MVA between $1 \mathrm{~cm}^{2}$ and $1.5 \mathrm{~cm}^{2}$, and no agreement at MVA more than 1.5 $\mathrm{cm}^{2}$. Moreover, after determining MVA by 3D methods, patients with MVA> $1.5 \mathrm{~cm}^{2}$ were excluded from planned PTMC.

Traditionally, 2D-TTE is a well-established routine method for MVA measurement in the case of patients with mitral stenosis, which is believed to be unaffected by hemodynamic changes. However, considering that obtaining an optimal perpendicular short-axis plane that crosses the tip of the mitral leaflets is sometimes difficult, the 2D-TTE method is less accurate than the 3D-TEE. Our results demonstrated that 
there was a significant correlation between MVA measured by 2D-TTE and 3D-TEE methods at MVA less than $1.5 \mathrm{~cm}^{2}$, but at MVA above $1.5 \mathrm{~cm}^{2}$ the correlation is non-significant. This results showed that 2DTTE has acceptable and consistent results and could be used confidently for planning patients with severe MS for PTMC but in the case of patients with discrepancy between clinical and echocardiographic findings, 3D-MPR or 3D-direct could be used for decision making. According to the current guidelines, an accurate determination of MVA is essential for choosing the best therapeutic strategy for MS patients (16).

Our results were supported by the findings of some previous studies $(17,18)$. Min et al. compared the MVA measured by 2D-TTE and 3D-TEE in 87 patients with MS. Our results were contrary to this essay. They reported more overestimation in the measurement of the MVA by $0.19 \pm 0.2 \mathrm{~cm}^{2}$ with 2D-TEE in comparison to 3D-MPR. On the other hand, our study showed that 2D-TTE underestimates MVA compared to 3D-MPR, but overestimates when it is compared to 3D-direct.

Mean MV gradient had no significant correlation with MVA measured by both 2D-TTE and 3D-TEE methods in our study. Likewise, in the study of Najih et al. $42 \%$ of patients with severe MS (MVA $\left.<1 \mathrm{~cm}^{2}\right)$ had a mean MV gradient $<10 \mathrm{mmHg}$, which suggests the absence of a direct correlation between an MVA $<1 \mathrm{~cm}^{2}$ or $<1.5 \mathrm{~cm}^{2}$ and a mean MV gradient $>10 \mathrm{mmHg}$ (19). It was notable that even severe MS exists with a mean MV gradient $<10 \mathrm{mmHg}$ (19). The 2020 European Society of Cardiology guideline defined the severe MS as an MVA of $<1.5 \mathrm{~cm}^{2}$ and a mean MV gradient of $>5 \mathrm{mmHg}$, under a condition in which this gradient is interpreted as a product of the heart rate and the patient having sinus rhythm (20). Consequently, although the mean MV gradient is an important indicator of MS tolerance, it is not a reliable marker of MS severity. This is mostly because of its dependence on several hemodynamic parameters including rhythm, heart rate, cardiac output, and the coexistence of mitral insufficiency $(5,20$, 21). Thus, it is suggested that the value of the mean MV gradient should never be interpreted as a single value.

The systolic PAP estimated through echocardiography had a significant inverse correlation with MVA in all measurement methods; therefore, it seems to be better than MG in assessing the severity of mitral valve stenosis.

AF rhythm was found in $44.3 \%$ of the patients which warrants anticoagulation.

\section{Conclusion}

MVA measurement by 3D-direct method showed generally significant consistency and agreement with 3D-MPR method especially at MVA less than $1.5 \mathrm{~cm}^{2}$. In practice, 3D-direct could be an acceptable and relatively less time-consuming method for measuring MVA. 2D-TTE planimetry yields an acceptable accuracy in measurement of MVA, comparable to that of 3D-MPR and 3D-direct planimetry, but its correlation with 3D-TEE methods is significantly reduced in cases where the valve stenosis is moderate (i.e. MVA> 1.5cm2). 2D-TTE and 3D-direct TEE methods slightly underestimated MVA compared to 3D- 
MPR, especially at MVA above $1.5 \mathrm{~cm}^{2}$. It's better to Carefully interpret the results of the latter two methods in conjunction with 3D-MPR. Incomplete evaluation of mitral valve commissures area due to saddle-like shape of MV at progressive (moderate) MS is probably a cause of 3D-direct underestimation. Finally, MPG failed to show a significant correlation with MVA measured by both 2D-TTE and 3D-TEE methods. Pulmonary artery systolic pressure had a significant inverse relation with measured MVA.

\section{Limitations Of The Study}

Our study had some limitations that should be addressed. It could be preferable to assess the 3D-TTE results and compare them with the results of 3D-TEE and 2D-TTE. However, the main aim of this study was to evaluate the accuracy of the results of different methods of 3D-TEE planimetry in patients with severe MS. Another limitation is the lack of invasive hemodynamic results related to MG and SPAP, which could have been helpful in assessing the true gradient and pulmonary pressure.

\section{References}

1. Ranjan R, Pressman GS (2018) A etiology and epidemiology of mitral stenosis. E-Journal of Cardiology Practice 16:14

2. Manjunath CN, Srinivas P, Ravindranath KS, Dhanalakshmi C (2014) Incidence and patterns of valvular heart disease in a tertiary care high-volume cardiac center: a single center experience. Indian Heart J 66(3):320-326

3. Reményi B, Wilson N, Steer A, Ferreira B, Kado J, Kumar K et al (2012) World Heart Federation criteria for echocardiographic diagnosis of rheumatic heart disease-an evidence-based guideline. Nature reviews Cardiology 9(5):297-309

4. Nishimura RA, Carabello BA, Faxon DP, Freed MD, Lytle BW, O'Gara PT et al (2008) ACC/AHA 2008 guideline update on valvular heart disease: focused update on infective endocarditis: a report of the American College of Cardiology/American Heart Association Task Force on Practice Guidelines: endorsed by the Society of Cardiovascular Anesthesiologists, Society for Cardiovascular Angiography and Interventions, and Society of Thoracic Surgeons. Circulation 118(8):887-896

5. Baumgartner H, Hung J, Bermejo J, Chambers JB, Evangelista A, Griffın BP et al (2009) Echocardiographic assessment of valve stenosis: EAE/ASE recommendations for clinical practice. $J$ Am Soc Echocardiogr 22(1):1-23

6. Baumgartner $\mathrm{H}$, Hung J, Bermejo J (2009) Echocardiographic assessment of valve stenosis: European Association of Echocardiography (EAE)/American Society of Echocardiography (ASE) recommendations for clinical practice. Eur J Echocardiogr 10:1-25

7. Dreyfus J, Brochet E, Lepage L, Attias D, Cueff C, Detaint D et al (2011) Real-time 3D transoesophageal measurement of the mitral valve area in patients with mitral stenosis. European journal of echocardiography 12(10):750-755 
8. Messika-Zeitoun D, Brochet E, Holmin C, Rosenbaum D, Cormier B, Serfaty J-M et al (2007) Threedimensional evaluation of the mitral valve area and commissural opening before and after percutaneous mitral commissurotomy in patients with mitral stenosis. European heart journal 28(1):72-79

9. Schlosshan D, Aggarwal G, Mathur G, Allan R, Cranney G (2011) Real-time 3D transesophageal echocardiography for the evaluation of rheumatic mitral stenosis. JACC Cardiovascular imaging 4(6):580-588

10. Anwar AM, Attia WM, Nosir YF, Soliman OI, Mosad MA, Othman M et al (2010) Validation of a new score for the assessment of mitral stenosis using real-time three-dimensional echocardiography. $J$ Am Soc Echocardiogr 23(1):13-22

11. Toufan M, Khezerlou N, Faraji H. Non inferior results in direct mitral valve planimetry with three dimensional(3D) echocardioghraghy compared to multiplanar reconstruction (MPR). European Heart Journal Cardiovascular Imaging. 2019

12. Zhong X, Chen W, Shi Z, Huan Z, Ma L, Liu W et al. Three-dimensional transesophageal echocardiography measurement of mitral valve area in patients with rheumatic mitral stenosis: multiplanar reconstruction or 3D direct planimetry?. The international journal of cardiovascular imaging. 2020.

13. Sadeghian H, Rezvanfard M, Jalali A (2019) Measurement of mitral valve area in patients with mitral stenosis by 3D echocardiography: A comparison between direct planimetry on 3D zoom and 3D quantification. Echocardiography 36(8):1509-1514

14. Nishimura RA, Otto CM, Bonow RO, Carabello BA, Erwin JP, Fleisher LA et al (2017) 2017 AHA/ACC focused update of the 2014 AHA/ACC guideline for the management of patients with valvular heart disease: a report of the American College of Cardiology/American Heart Association Task Force on Clinical Practice Guidelines. J Am Coll Cardiol 70(2):252-289

15. Eibel S, Turton E, Mukherjee C, Bevilacqua C, Ender J (2017) Feasibility of measurements of valve dimensions in en-face-3D transesophageal echocardiography. Int J Cardiovasc Imaging 33(10):1503-1116

16. Otto CM, Nishimura RA, Bonow RO, Carabello BA, Erwin lii JP, Gentile F et al (2021) 2020 ACC/AHA guideline for the management of patients with valvular heart disease: a report of the American College of Cardiology/American Heart Association Joint Committee on Clinical Practice Guidelines. J Am Coll Cardiol 77(4):e25-e197

17. Schlosshan D, Aggarwal G, Mathur G, Allan R, Cranney G (2011) Real-time 3D transesophageal echocardiography for the evaluation of rheumatic mitral stenosis. JACC: Cardiovascular Imaging 4(6):580-588

18. Min S-Y, Song J-M, Kim Y-J, Park H-K, Seo M-O, Lee M-S et al (2013) Discrepancy between mitral valve areas measured by two-dimensional planimetry and three-dimensional transoesophageal echocardiography in patients with mitral stenosis. Heart 99(4):253-258 
19. Najih H, Arous S, Laarje A, Baghdadi D, Benouna MG, Azzouzi L et al (2016) [Discordance between mitral valve area (MVA) and pressure gradient in patients with mitral valve stenosis: mean transmitral valve gradient is a severity index or a tolerance index of severity of mitralss valve stenosis]. Pan Afr Med J 25:75

20. Vahanian A, Alfieri O, Andreotti F, Antunes MJ, Barón-Esquivias G, Baumgartner $\mathrm{H}$ et al (2012) Guidelines on the management of valvular heart disease (version 2012). Eur Heart J 33(19):24512496

21. Nishimura RA, Otto CM, Bonow RO, Carabello BA, Erwin JP 3rd, Guyton RA et al (2014) 2014 AHA/ACC Guideline for the Management of Patients With Valvular Heart Disease: executive summary: a report of the American College of Cardiology/American Heart Association Task Force on Practice Guidelines. Circulation 129(23):2440-2492

\section{Figures}




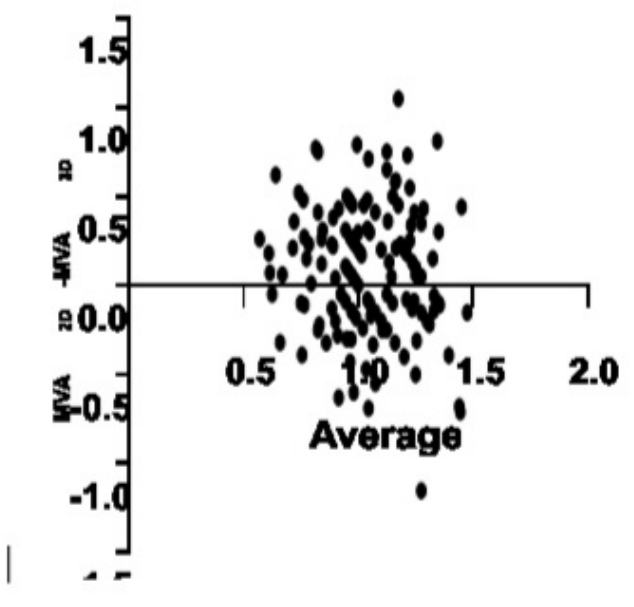

A

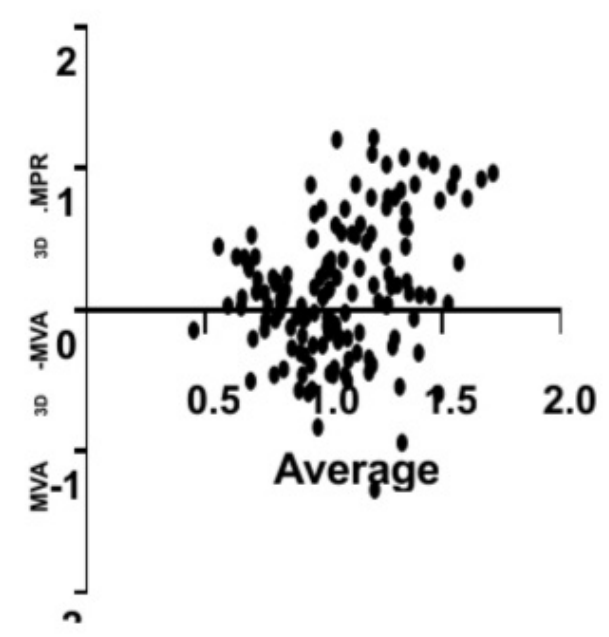

C

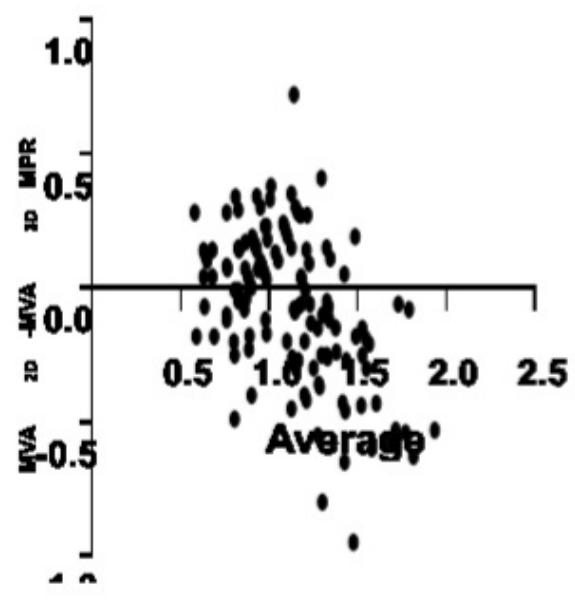

B

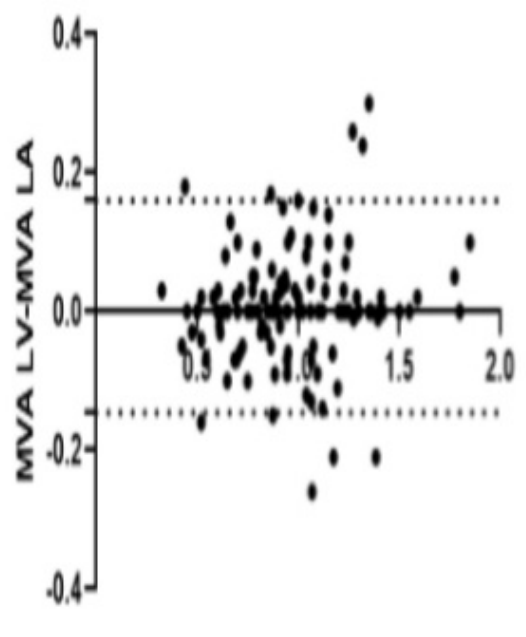

D

\section{Figure 1}

Bland-Altman compression between mitral valve area measured by different echocardiographic methods. The analysis of the data revealed that: A) MVA measured by 3D-direct method and 2D-TTE had a significant consistency with each other (Intraclass Correlation $=0.787$, $p$ value $=0.001$ ). B) MVA measured with 3D -MPR method was significantly correlated with the MVA measured by 2D-TTE with a moderate agreement (Intraclass Correlation $=0.644$, p value $=0.001) . C$ ) MVA measured by 3D-direct had 
a statistically significant agreement with that measured by 3D-MPR (Intraclass Correlation $=0.716, p$ value $=0.001)$. D) MVA measured by 3D-direct through LA and LV views were statistically in concordance (Intraclass Correlation $=0.964, \mathrm{p}$ value $=0.001)$.

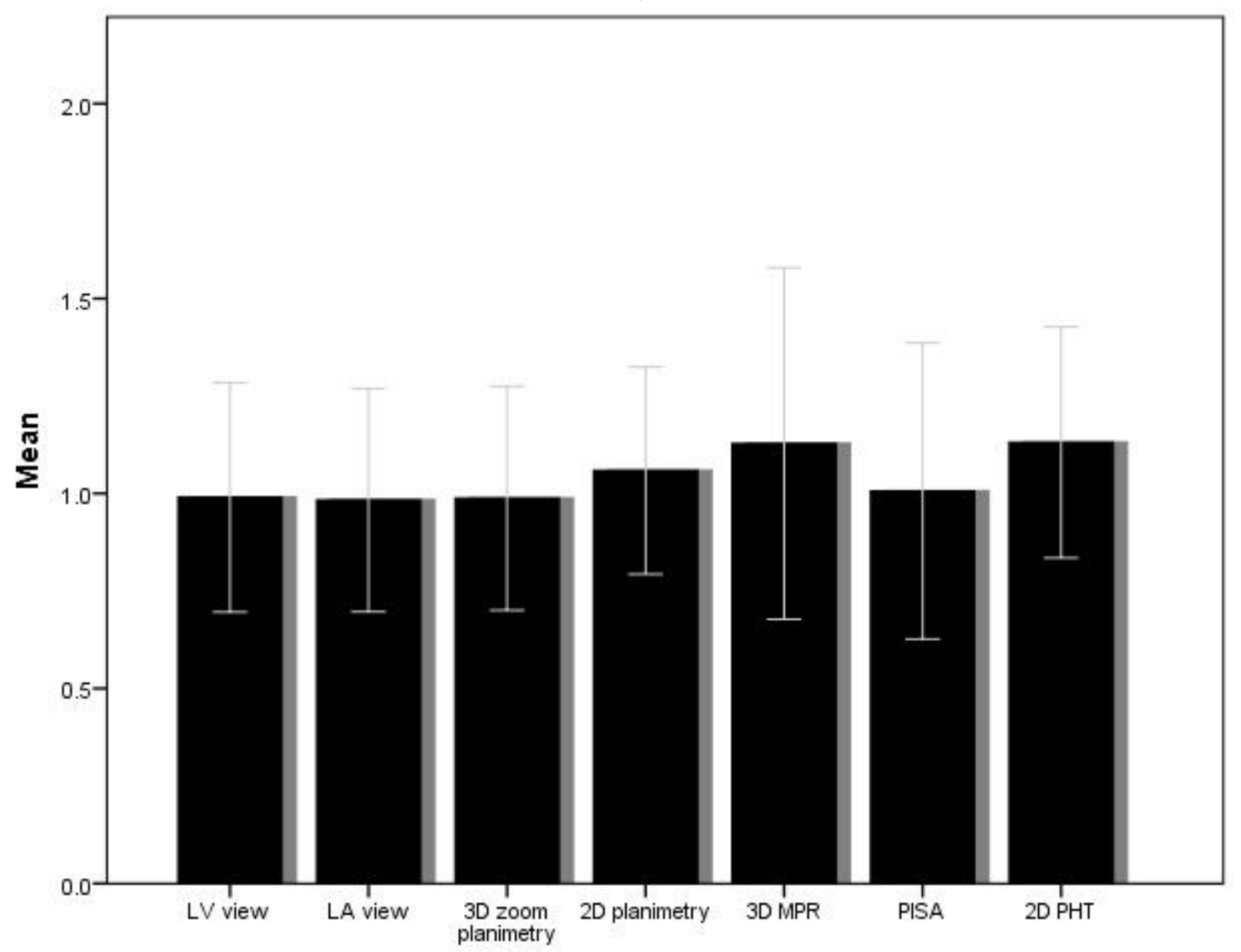

Error Bars: +/- $1 \mathrm{SD}$

Figure 2

MVA measurement with different echocardiographic methods. 


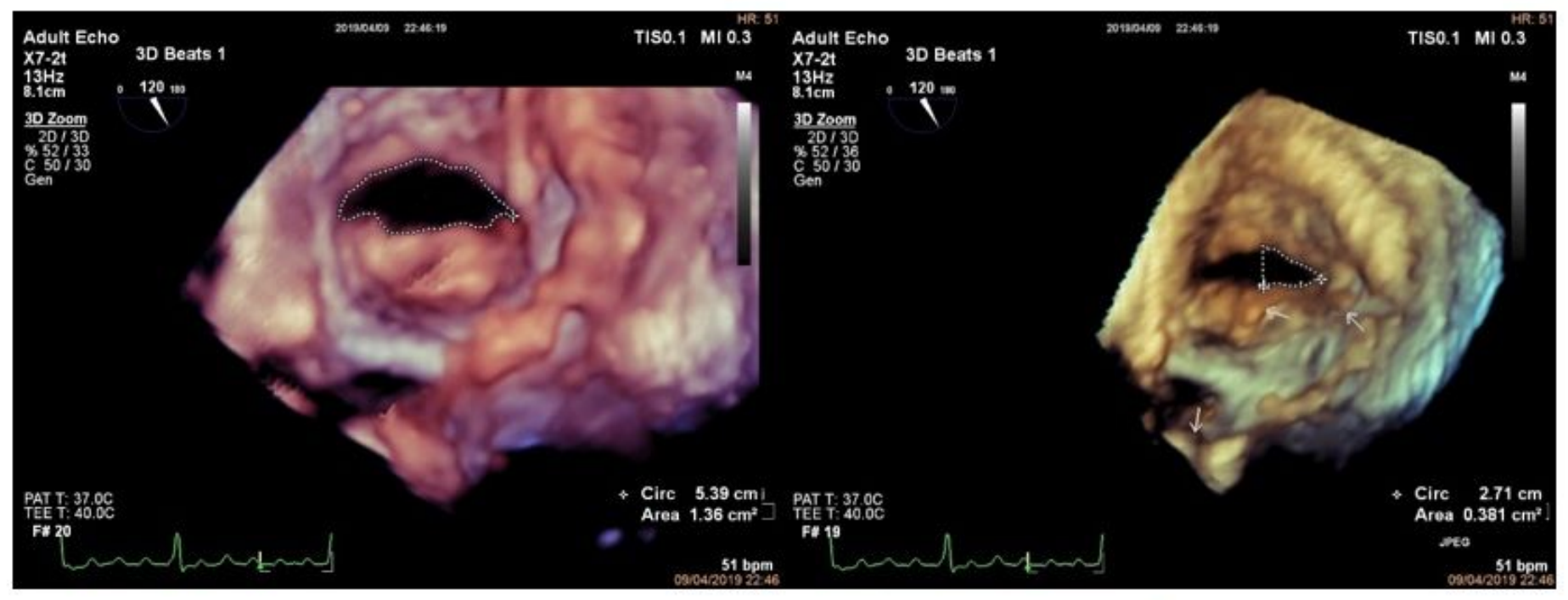

(a)

(b)

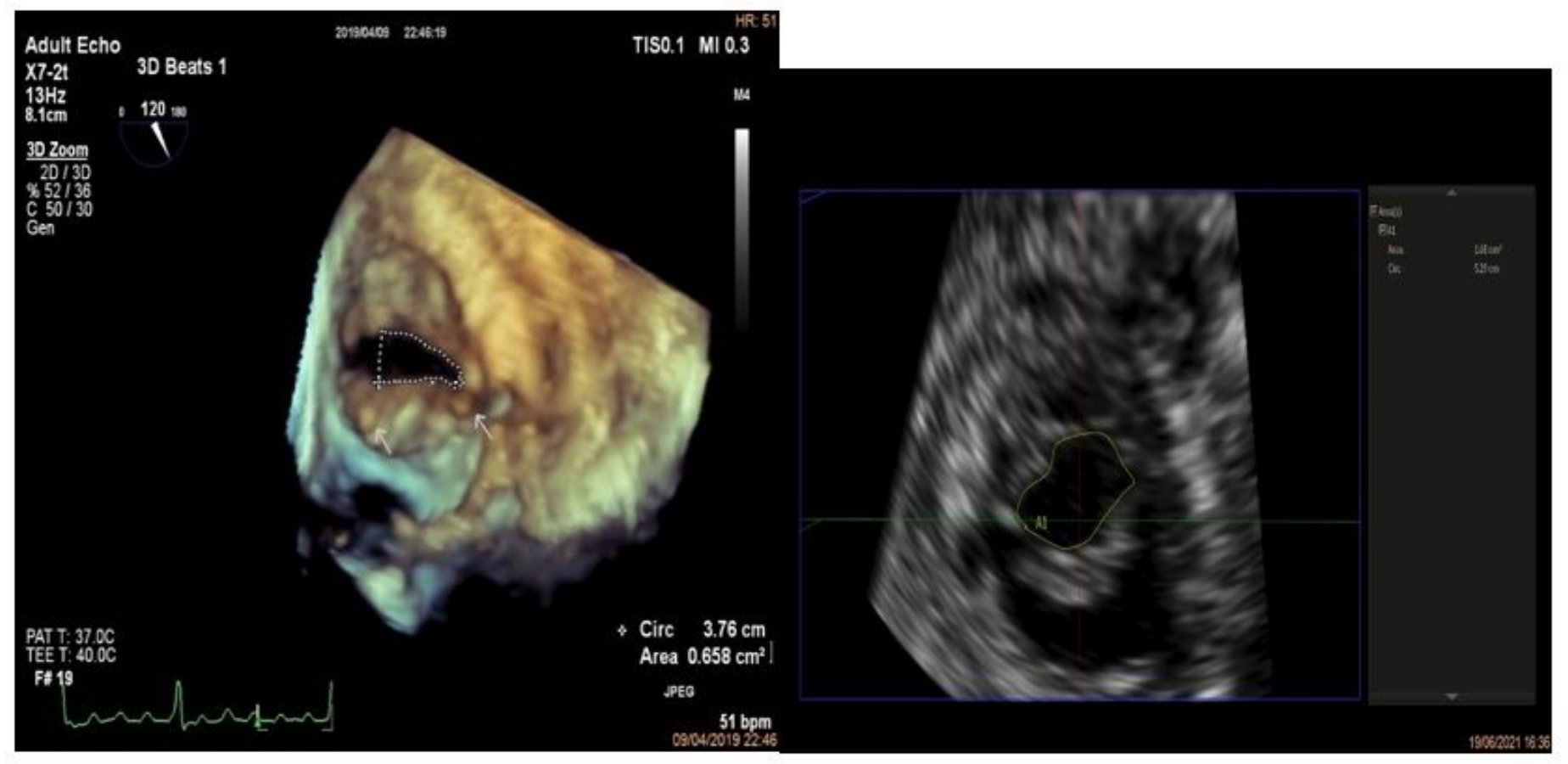

(c)

(d)

\section{Figure 3}

A comparison of MVA measurement with 3D-direct and 3D-MPR methods in a patient with moderate MS and the impact of commissural area measurement. A. Measurement of entire MVA with 3D-direct method $(1.36 \mathrm{~cm} 2)$. B and C. Different results of measuring the area of the same portion of MVA by 3D-direct method between the lateral commissure and the constant anatomical region marked with white arrows; at the en face view $(0.38 \mathrm{~cm} 2)(B)$ and at the oblique view $(0.65 \mathrm{~cm} 2)(C)$ which shows the unequal values 
in the two different oriented views in a single commissural area. D. Measurement of entire MVA with 3DMPR method in the same patient (D).

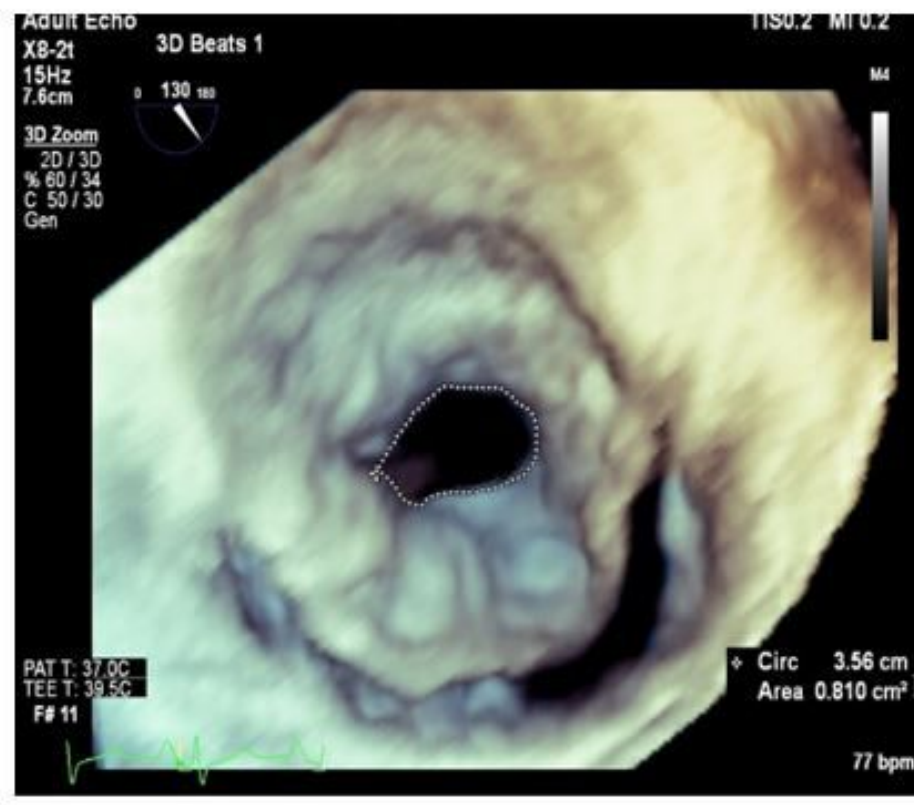

A

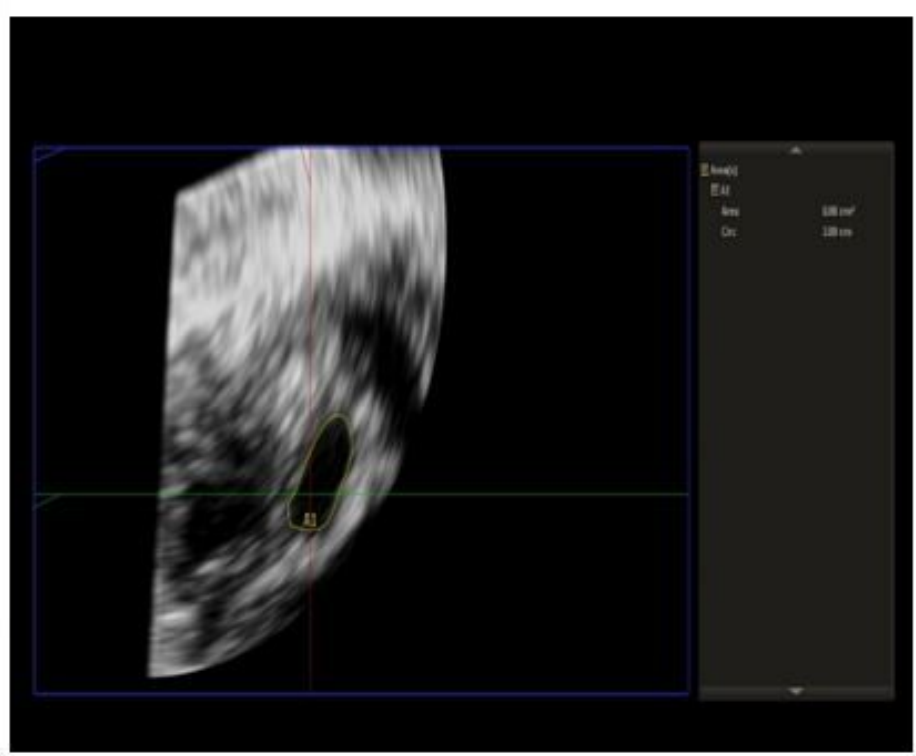

B

\section{Figure 4}

A comparison of MVA measurement by 3D-direct $(0.81 \mathrm{~cm} 2)$ and 3D-MPR $(0.88 \mathrm{~cm} 2)$ methods in a patient with severe MS. 